\title{
Tangence
}

\section{Sous le signe de l'absence : une mise à l'épreuve du programme mythique de l'année 1968 au Québec}

\section{Hélène Marcotte}

Numéro 50, mars 1996

Lectures de nouvelles québécoises

URI : https://id.erudit.org/iderudit/025897ar

DOI : https://doi.org/10.7202/025897ar

Aller au sommaire du numéro

Éditeur(s)

Tangence

ISSN

0226-9554 (imprimé)

1710-0305 (numérique)

Découvrir la revue

Citer cet article

Marcotte, H. (1996). Sous le signe de l'absence : une mise à l'épreuve du programme mythique de l'année 1968 au Québec. Tangence, (50), 131-141.

https://doi.org/10.7202/025897ar d'utilisation que vous pouvez consulter en ligne.

https://apropos.erudit.org/fr/usagers/politique-dutilisation/ 


\title{
Sous le signe de l'absence: une mise à l'épreuve du programme mythique de l'année 1968 au Québec
}

\author{
Hélène Marcotte
}

\author{
J'ai mal \\ au passé \\ mort \\ au présent \\ absent \\ de demain \\ Madeleine Guimont, \\ Les temps miscibles
}

Les années 1966 à 1969, au Québec, semblent avoir souffert du prestige accordé à la Révolution tranquille et aux événements d'Octobre 1970. Pourtant, les nombreux bouleversements qui se produisent alors laissent croire que ces années s'inscrivent comme un point tournant dans l'histoire du Québec. L'année 1968, plus particulièrement, "apparaît comme une année char-

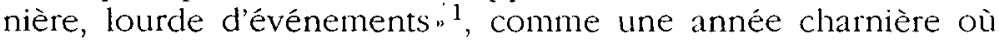
rupture, continuité et renouveau se côtoient. Dans la vie politique et sociale, on assiste, entre autres, à la victoire du Parti libéral aux élections fédérales, avec Pierre Elliott Trudeau comme chef, à la formation du Parti québécois et à la vague de contestation dans les cégeps et les universités. La vie culturelle et littéraire est marquée, pour sa part, par la création de plusieurs revues, comme Études littéraires, Les berbes rouges, de même que par la reprise de la Rencontre des écrivains après six ans d'interruption, la fondation du Centre du Théâtre d'aujourd'hui, la publication du manifeste-agi "Place à l'Orgasme" par l'Opération Déclic, les premiers spectacles des "Poèmes et Chants de la résistance", "l'Osstid'cho" et la création des Belles-sceurs de Michel Tremblay, pour ne citer que les événements les plus importants. Bref, c'est

1 Dorval Brunelle, "De la Révolution tranquille à la chute de 13ourassa "Le Québec en textes. Anthologie 1940-1986, Montréal, Éditions Boréal, 1986, p. 180 . 
132

l'effervescence dans tous les domaines. Les esprits, en ébullition, poursuivent un même but : changer le monde.

Toutes ces métamorphoses, tous ces mouvements de masse attirent l'attention sur cette période de l'histoire du Québec où s'inscrivent les manifestations d'un esprit nouveau. Dans des recherches précédentes ${ }^{2}$, nous avons étudié l'année 1968 en prenant comme objet d'analyse la production littéraire parue au Québec au cours de cette année et, comme méthode de lecture, celle que propose la mythanalyse, à savoir, d'après la définition de Gilbert Durand: "l'analyse des mythes en tension dans une certaine société, à une certaine époque" ${ }^{3}$. Nous avons essayé d'entrevoir le jeu dynamique par lequel les poètes agencent leurs angoisses, leurs désirs et leurs visions du monde, bref, de cerner le programme mythique de l'année 1968 au Québec. Comme le propose Jacques Blais:

Ce programme mythique réunit des éléments en provenance de plusieurs mythes ou légendes provisoirement interreliés et vécus comme olssédiants durant lá période à l'étucle. Par-clelì la superposition de ces indices pourrait en définitive se révéler, tel un palimpséste, le récit archétypal auquel tenclent plus ou moins de s'ajuster les textes et les événements de la période concernée. ${ }^{4}$

Selon notre hypothèse de travail, le programme mythique de l'année 1968 au Québec comporte quatre thèmes: les thèmes du Double, de la révolte, de la quête et de la nouvelle naissance. À chacun de ces thèmes sont associées des figures mythiques qui en actualisent les traits dominants: il s'agit, pour le Double, de Narcisse; pour la révolte, de Dionysos; pour la quête, de Jason (le chef des Argonautes); et, pour la nouvelle naissance, d'Adam et Ève.

Le mythe de Narcisse, tel que pressenti dans les oeuvres littéraires, prend toute sa signification quand on le replace dans la

2 Nous n'avons pas analysé tous les ouvrages parus au Québec au cours de l'année 1968. En fait, nous avons étudié plus particulièrement une dizaine de recueils de poésie (voir Hélène Marcotte, "L'heure d'hier et de demain. Mythanalyse de la poésie québécoise en 1968 , mémoire de maîtrise ès arts, Québec, Université Laval, 1988, 120 f.), de mêtne que certains romans et quelques pièces de thêâtre.

3 Gilbert Durand, L'âme tigrée, Paris, Denoèl/Gonthier, 1980, p. 152.

4 Jacques Blais, "Mithra/Malicroix : le roman de Bosco et les mythes de l'immédiat après-guerre (1945-1950)», Études littéraires, vol. XVII, $\mathrm{n}^{\circ} 1$, avril 1984 . p. 48 . 
perspective de l'individu qui se sent dépossédé et impuissant face aux événements qui surviennent. Le sentiment de dépossession s'exacerbe jusqu'à la perte de l'identité et l'avènement du Double. La révolte dionysiaque, pour sa part, ordonne les bouleversements survenus selon les traits les plus accusés de ce mythe, soit le jeu thêâtral, la fête, la folie et la violence. La résurgence de Dionysos témoigne du désir d'émancipation et de libération qui hante les individus, la violence n'étant qu'un moyen de s'affranchir d'une situation intolérable et de parvenir à la résolution du Double. La destruction symbolique du monde, ou tout simplement de l'Autre, doit se réaliser pour que soit possible la nouvelle naissance. Placée sous le signe de Jason, la quête rend compte des diverses tentatives ébauchées pour recréer un ordre nouveau de même que des obstacles rencontrés. Cette quête, qui prend souvent l'allure d'un quelconque voyage, se traduit par une recherche du pays et de l'amour, de même que par une recherche de l'écriture et de la parole. Cependant, toute l'énergie déployée pour atteindre les objectifs de la quête ne suffit point. Une brève période d'euphorie fait parfois croire à l'aube de jours meilleurs, mais cette vie à outrance se révèle éphémère. En fait, la nouvelle naissance est reportée à demain, à plus tard, voire à jamais.

Comme il est fort possible, pour ne pas dire probable, que l'interaction des quatre mythes identifiés ne coïncide pas uniquement avec l'année 1968, mais domine l'imaginaire québécois durant une période plus étendue, la seconde étape de notre recherche consiste à confronter le programme mythique, tel qu'esquissé par l'analyse de la production littéraire de l'année 1968 , au contenu des autres textes publiés au cours des années 1966 à 1969. Dans le cadre de cet article, nous avons donc choisi deux nouvelles, soit "De retour le 11 avril" de Hubert Aquin et "Un parfum de rose bleue" de André Langevin ${ }^{5}$. Ces nouvelles figurent parmi celles qui ont été commandées, en 1967, par Radio-Canada à douze auteurs (six écrivains du Québec et six écrivains dụ Canada anglais), et publiées en 1969 dans un numéro spécial de la revue Liberté, intitulé Douze écrivains. Douze nouvelles.

5 Hubert Aquin, De retour le 11 avril *, Liberté, mars-avril 1969, p. 5-19 et André Langevin, "Un parfum de rose bleue *, ibid., p. 61-75. 
134

\section{L'obsession de la mort}

La nouvelle de Hubert Aquin prend la forme d'une longue lettre d'adieu, rédigée "sans passion et sans grande émotion" (p. 8) par le narrateur au moment même où il avale "plus de capsules qu'il n'en faut pour mourir" (p. 19). S'adressant à la femme aimée, le narrateur évoque tout au long de cette lettre les derniers mois de sa vie, passés en solitaire. Car chez Aquin, la dépossession est liée à la perte de l'être aimé :

Jaimerais bien, moi aussi, pouvoir nier ce long hiver qui n'en

finit plus, par sa froide agonie, de me rappeler les mois neigeux que j'ai vécus sans toi, loin cle toi. (p. 7)

Ce retour que le personnage effectue sur son passé, par le biais de l'écriture, fait surgir l'image de Narcisse, symbole tragique de l'être dédoublé, écartelé, voire écarté de son destin. Ici, le Double naît de la division de l'être qui prend à la fois conscience de son existence et de son inexistence à travers l'image qu'il entrevoit de lui-même, mais aussi et surtout à travers l'image de la femme aimée, image qui risque à tout moment de disparaître ou de l'absorber, le condamnant, dans les deux cas, à une mort certaine. Le protagoniste mesure soudain "la distance entre le monde réel et le monde désiré, entre la volonté du Moi et la réalité de son destin " 6 . Tous les possibles qu'il avait espéré voir se concrétiser durant les premiers temps de sa relation semblent relégués aux oubliettes, et le fol espoir qui l'avait alors soulevé s'évanouit. Le réveil n'en est que plus brutal, la désillusion, plus amère, et la longue lettre d'adieu trahit le désarroi qui s'ensuit.

L'éloignement de la personne aimée et la solitude qui en résulte n'éveillent guère de mouvement de révolte chez le protagoniste. En fait, toute la violence est dirigée contre lui-même, tandis que la quête se réduit à trouver un moyen de mettre fin à ses jours. L'idée de la mort seule semble guider les gestes du narrateur, le présent oscillant sans cesse entre hier et demain, entre le début de l'agonie et la lente mort, semblable à un sommeil sans fin. Mais intimement lié à cette image obsédante de la mort, se glisse l'espoir d'échapper à ce destin tragique ou, à tout le moins, d'en retarder l'issue fatale:

6 En collaboration, Le double dans le romantisme anglo-américain, Faculté des lettres et sciences humaines de l'Université de Clermont-Ferrand II, fascicule 19, 1984, p. 31. 
En quelque sorte, je savais que jallais mourir au moment voulu et je devais maccommoder de ce projet funèbre tout en attenclant - qui sait un mot de toi, une lettre, ton retour... Plusieurs fois par jour, j’allais ouvrir la petite boite postale, le cour battant, dans l'espoir que $j y$ trouverais la raison inespérée de t'attendre. (p. 12)

Dans cette perspective, l'écriture de la lettre d'adieu se veut un apprivoisement de la mort plutôt qu'un prolongement de la douleur. Le narrateur ne cherche pas à condamner qui que ce soit ou à se plaindre; il pose plutôt un regard clinique sur les derniers événements et annonce qu'il n'attend "plus rien du printemps" (p. 18). L'écriture repousse momentanément non seulement la mort mais aussi la souffrance qui devient, sous la plume de l'épistolier, le récit d'une souffrance. Elle permet une mise à distance et le passé apparaît moins sur le mode tragique que comme le simple constat d'un échec amoureux.

Après l'absorption des comprimés, le narrateur note: "Franchement, je n'étais pas triste, mais impressionné comme celui qui part pour un long voyage. (p. 13). Comme cette comparaison suggère un commencement plutôt qu'une fin, le lecteur peut déjà soupçonner l'échec de cette première tentative, si l'écriture même de la lettre ne le lui avait pas déjà annoncé. Puis le héros se réveille sur un lit d'hôpital. Toutefois, ce réveil, loin d'être comparé à une renaissance, est vécu comme un retour à la situation initiale: "Dehors, il neigeait à nouveau, comme à la veille de ton départ [...] La neige tombait dehors; je vivais, mais toi où étais-tu?" (p. 15). Le narrateur ne se dit pas tant ranimé par l'équipe médicale que "prolongé dans [s]on agonie, perpétué dans [s]a faiblesse et [s]on impuissance". "[J]e valais tout juste mon prix net en viande dégénérée, plus la facture d'hôpital " 7 , constate-t-il (p. 17). Obligé, bien malgré lui, de renoier avec la vie, il emploie ses nouvelles forces à "[s]e couvrir de nuit" (p. 19). Dans la nouvelle de Hubert Aquin, la nouvelle naissance ne peut se réaliser:

7 La définition de l'homme québécois de 1968 que propose un tract distribué par l'université libre d'art quotidien (ULAQ) met l'accent à son tour sur l'animalité de l'homme en le décrivant comme *un paquet numéroté de viande saignante conditionné obligatoirement à fabriquer de la marchandise [...] Yves Robillard (dir.), Québec underground. 10 ans d'art marginal au Quéhec, 1962-1972, Montréal, Éditions Médiart, 1973, tome 2, p. 109. 
136

Pour moi, la nuit finale allait commencer - une seule et même nuit qui ne finirait plus, une longue nuit qui mettrait un terme à notre histoire désordonnée, à la longue hésitation, à ta sincérité embrouillée et à tes intermittences - tout cela de toi qui n'avait été cruel que dans la mesure où j'en avais souffert. (p. 13)

La quête de l'amour est vaine, le paysage se recouvre de neige, et la parole, ou plus précisément l'écriture, renvoie l'être à son néant: "Les silences entre les mots, sont autant de pores par lesquelles j'absorbe mon propre néant " (p. 19). À la fin du récit, au moment de glisser dans la mort, la narrateur revit, en quelque sorte, le moment de la rupture, lors même qu'il neigeait dans son coeur comme il neigeait sur la ville, et lance un dernier cri vers l'aimée: "Il neige en moi doucement, sans arrêt, de plus en plus, et j'ai froid. Mon amour, j'ai froid..." (p. 19). Malgré la présence sans cesse rappellée du destinataire et l'évocation d'un passé commun, "De retour le 11 avril" se veut le récit d'un rendez-vous manqué.

\section{Le parfum de l'impossible}

Dans «Un parfum de rose bleue" d'André Langevin, enfermés au douzième étage d'un immeuble, deux hommes discutent au sujet d'une femme décédée. L'identité de cette dernière paraît problématique. Non seulement elle est prénommée Jeanne par l'un, le mari, et Anne par l'autre, l'amant d'une nuit, mais la photo reproduite dans le journal ne coïncide pas vraiment avec l'image qu'en garde l'amant:

- Anne ou Jeanne, cela n'a aucune importance. Vous l'avez reconnue? C'est bien sa photo?

- Oui et non. Anne avait un autre regard, une autre façon de sourire, une passion. (p. 64)

C'est donc le double regard posé sur la jeune femme qui met en relief son dédoublement et la fragilité de son identité. Le lecteur ne parvient à connaître que quelques fragments de sa vie à travers les brèves évocations que s'arrachent les deux hommes et qui, de plus, se contredisent, rendant le réel fuyant et incertain.

À l'origine du périple de Jeanne/Anne se retrouve le sentiment de dépossession: "Je dois partir, une semaine ou deux, ou plus longtemps encore. J'étouffe. Vous respirez mon air (p. 65), écrit-elle à la hâte sur un papier avant de s'enfuir. Le sentiment 
de dépossession de la jeune femme se révèle toutefois nécessaire puisqu'il précède, prépare et provoque le désir de réappropriation, d'unification et de libération. L'héroïne souhaite reprendre son destin en main et, presque simultanément à la prise de conscience de la dépossession s'amorcent la révolte et la quête.

Celui qui deviendra l'amant de Jeanne/Anne fait sa rencontre dans la rue, alors qu'elle vient de perdre l'équilibre et qu'elle patauge dans la boue. Il lui offre alors de venir se sécher chez lui et l'invite à souper. La jeune femme profite de son hospitalité pendant douze heures:

Durant ces quelques heures, Anne a été sans passé et sans avènir, elle-même ou une autre, je ne sais, Jeanne ou Anne, liée à cet homme ou à un autre, elle a été un appel fait à moi seul, une inconnue inoubliable. (p. 67)

La relation qui se noue entre les deux futurs amants n'est pas sans évoquer le jeu théâtral, un des éléments structurants du mythe de Dionysos:

Ainsi avait clébuté une nuit très lente, très dọce, sans cesse menacée d'une rupture imprécise, parce que la part du jeu y était totale. Aucun de nous n'a cherché à savoir qui était l'autre. Nous nous donnions l'un à l'autre une représentation qui était la vérité du moment, et rien d'autre ne comptait. (p. 72)

Par ce jeu, la parole est efficace sitôt dite et s'ancre dans l'immédiat puisqu'elle crée la réalité plus qu'elle ne cherche à la traduire. Le jeu devient un refuge momentané. Si le recours à l'imaginaire peut être révélateur d'une perte ou d'un manque, il peut être aussi créateur de sens. Ainsi, la fuite de Jeanne/Anne prend la forme de la fiction, et l'aventure des amants se déroule en marge de la réalité, du quotidien, dans un ailleurs qui se veut davantage vertige que folie, oasis qu'exil. La musique de Mozart qui envahit l'appartement au moment de l'acte d'amour participe de cet arrachement au monde réel et isole les amants dans une intimité magique et précaire. Mais ni le rêve ni l'amour ne sont plus possibles pour Jeanne/Anne et l'acte d'amour ne peut se comparer ici à une renaissance. Les amants se révèlent incapables de se rejoindre véritablement et de façon durable. Jeanne/Anne demeure une énigme, "si lointaine quand elle me tendait les bras" (p. 64).

La création du personnage "Anne" est en outre attestée par l'invention d'un passé qui prend le contre-pied de la véritable his- 
toire de Jeanne, du moins telle qu'elle nous est rapportée par le mari :

- J'ai tué un ours autrefois. Ces bêtes énormes meurent comme de tout petits enfants, sans résister. Je ne chasse plus.

- Une simple sauterelle sur la pelouse la terrorisait.

- Je n'ai rien éprouvé quand mes parents sont morts. Ils étaient si tranquilles...

- Ils vivent encore [...]. (p. 69)

La jeune femme se crée une identité, un passé et peut-être même une passion. Alors que pour le mari cette fiction peut se comparer à une trahison, pour l'amant, le dire n'était pas un leurre et Anne reste pour lui plus vraie que Jeanne.

Mais, Anne ne l'emporte qu'un court moment sur Jeanne. En effet, les paroles prononcées par Jeanne/Anne à son amant ne parviennent pas à s'assembler pour former un discours cohérent :

Tout ce que je viens de dire n'a aucun sens. Ces mots, Anne les a bien prononcés, de sa voix un peu éteinte, qui émergealit à peine du silence, bribes de son discours intérieur qui s'échappaient d'elle à son insu presque, mais sans cette voix, sans son regard vacillant dans la nuit, sans sa présence si légère et, en même temps, si grave, ces mots-là ne peuvent plus rien révéler. (p. 69-70)

Et qui plus est, * [n]on seulement ces mots n'ont plus de sens, mais de les avoir dits a effacé l'image d'Anne" (p. 70), constate l'amant. La réitération du passé, qui se traduit aussi par l'écoute commune du concerto en la pour clarinette de Mozart, altère la beauté des souvenirs de l'amant qui se sent peu à peu dépossédé à son tour. Il semble alors qu'au fur et à mesure que le mari se réapproprie Jeanne/Anne, il l'enlève à l'amant. À la fin du récit, le mari s'en va "[d]ans la paisible odeur du chou bouilli qui obnubile l'improbable parfum de rose bleue" (p. 75). Mais pour l'un comme pour l'autre, "une rose bleue serait le symbole de l'impossible ${ }^{8}$, impossible amour, impossible union des êtres, impossible réconciliation de l'être.

Comme on a pu le constater, le discours rapporté de Anne n'est pas sans suggérer la folie. Mais son discours, pour décousu

8 Jean Chevalier et Alain Gheerbrant, Dictionnaire des symboles, Paris, Rober Laffont/Jupiter, 1983, p. 824. 
qu'il soit, demeure intéressant pour ce qu'il suggère et poùr ce qu'il tait aussi. Ainsi, le rapport à autrui se révèle plus que problématique dans l'imaginaire de la jeune femme: elle choisit de faire mourir ses parents, de se présenter comme victime d'un viol ou encore comme grugée par la douleur des enfants du Vietnam, brûlés par le napalm. Ce pays semble d'ailleurs une véritable obsession chez elle: "C'est un pays chaud, dit-elle dans un long soupir. J'ai fait provision de froid pour plus d'une vie" (p. 73). Le lecteur obtient la clé de cette mystérieuse obsession dans les dernières lignes du récit, lorsqu'il apprend que le troisième enfant du couple, confié à un foyer nourricier dès sa naissance, est "un enfant asiatique dont le regard noir dévore le visage" (p. 75). Notons, enfin, que le rapport à soi est aussi vécu sur un mode négatif: "Je ne mange plus, je ne dors plus, et j'ai froid" (p. 73). Privée de toute énergie vitale, la jeune femme souffre tant dans son âme que dans son corps.

Dans "Un parfum de rose bleue", une seconde quête se superpose à la première. En effet, à la suite du décès de Jeanne, c'est son mari qui se sent dépossédé et qui cherche à élucider les derniers jours de la vie de sa femme: "Je cherche... comme un parfum, une respiration..." (p. 63). Il précise quelques instants plus tard: "Ce que je cherche ici... je ne sais pas. Une phrase qui, pour vous, n'a peut-être pas de sens, mais qui serait très précieuse pour moi " (p. 65). En fait, le mari s'efforce de comprendre -[p]ourquoi, sur une route déserte, parfaitement sèche, dans une voiture neuve, [elle] s'est [...] jetée à plus de cent à l'heure sur le seul arbre qu'il y avait à l'horizon. (p. 75). Tourné vers le passé, ce personnage ne tend pas, au terme de sa quête, vers une renaissance. Il demeure prisonnier de ses souvenirs et ne demande qu'à le demeurer.

\section{Sous le signe de l'absence}

Dans les deux nouvelles étudiées, les personnages tentent d'entrer en contact avec une autre personne par le biais de l'écriture, dans le premier cas, et par celui de la parole dans le second. Les multiples interrogations qui jalonnent les deux textes trahissent d'ailleurs le besoin qu'éprouvent les personnages de se saisir de l'autre. Pourtant, toujours ils tendent vers le silence. Dans la première nouvelle, les mots de l'aimée déçoivent sans cesse l'attente du héros qui, de son côté, n'écrit qu'une lettre 
140

d'adieu; dans l'autre texte, l'amant rapporte des phrases vides de sens :

Jai été violée dans une église par un enfant de chour. J'aime le parfum de l'encens. Je ne bois jamais de lait, parce que je n'aime pas le blanc. Je voudrais n'avoir que des enfants jaunes... Vous voyez bien qu'il n'y a rien à tirer de cela. (p. 69)

Et le jeu des questions-réponses ne fait que rendre l'identité de Jeanne/Anne plus problématique. En outre, le "nous" n'existe qu'au passé, comme pour bien souligner l'écart entre les êtres, la distance maintenue à jamais par les mots ou par la mort. Le passé apparaît comme un miroir déformant dans lequel l'image de la personne n'est plus qu'une image défaite et où l'être, croyant se retrouver, se perd. De refuge, le passé devient donc abîme.

La terre repousse le protagoniste et se couvre de neige ou de boue, l'amour s'éloigne et la voix s'éteint jusqu'à se faire silence. Pays, amour, parole, tout s'échappe et déserte les protagonistes. Ils se retrouvent tout à coup seuls, perdus et muets, ou plutôt délaissés, désorientés, bâillonnés. La sensation intense de froid qui les saisit et les paralyse traduit bien cette profonde solitude. Après la rupture amoureuse, le héros erre dans une étendue blanche, se heurte aux glaces, au givre, au froid, soudainement habité par un hiver sans nom. C'est peut-être pour cette raison qu'il préfère les lieux clos. Dans la nouvelle d'Aquin, dès qu'il sort, le protagoniste erre, se perd et ne sait que retourner sur les lieux chéris du passé: Il meurt d'ailleurs "réfugié dans une chambre d'hôtel, insonorisée et luxueuse comme un cercueil géant " (p. 19). Dans la seconde nouvelle, les espaces semblent plus vastes et plus ouverts, mais ils sont perçus aussi négativement parce qu'assimilés au froid. La désespérance qui s'ensuit ne trouve de limite que dans la mort. Dépossédés au départ, les personnages demeurent démunis au terme de leur recherche d'un meilleur état de vie. Tout reste donc à faire, à réaliser. Après l'ennui, l'errance, la solitude, les souffrances de toutes sortes et l'échec de la renaissance, les protagonistes décident d'inscrire le mot fin à leur histoire.

\section{Conclusion}

Comme on peut le constater, les quatre thèmes cernés par l'analyse de la production littéraire de l'année 1968 se retrouvent 
dans les nouvelles étudiées, mais avec des variantes et des nuances. Ainsi, les allusions mythologiques et les renvois aux figures mythiques, figures sur lesquelles les poètes de la fin des années soixante reviennent abondamment de façon à ne laisser aucun doute sur l'orientation et la signification données aux thèmes, sont beaucoup moins accusés. De plus, les thèmes de la révolte et de la quête sont si intimement liés dans les deux nouvelles qu'ils se confondent, voire s'euphémisent. La quête semble n'être qu'une préparation au suicide, geste qui marque à la fois son paroxysme et sa conclusion. Quant au suicide, qui confère simultanément au protagoniste le rôle de victime et de bourreaul, il ne met guère en relief l'idée de révolte. La violence envers soi, ici, se veut davantage synonyme de fuite. "Jeanne aimait se perdre" (p. 63), constate son époux, précisant un peu plus loin: "Jeanne n'était que fuite" (p. 65). La mort apparaît donc comme l'ultime refuge et les personnages renoncent à tout espoir. Les nouvelles se terminent par un constat d'échec et mettent en relief l'incapacité de l'individu de se lier à autrui de façon durable, de s'accorder avec son environnement ou encore de vivre en harmonie avec lui-même. Le rapport à soi passe nécessairement par autrui et de cette impossible réconciliation avec soi-même naît la désespérance. Plus que sous le signe de la souffrance, de la révolte ou de la mort, le récit commun, tel qu'ésquissé dans les ouvres étudiées, s'écrit sous le signe de l'absence. 